\title{
ACTITUDES, COMPORTAMIENTOS Y USOS DE DIFERENTES GENERACIONES DE USUARIOS EN INTERNET
}

\section{ATTITUDES, BEHAVIOURS AND USES OF DIFFERENT \\ GENERATIONS OF USERS IN INTERNET}

\begin{abstract}
AUTOR
Mónica Matellanes Lazo: Profesora Doctora Adjunta. Universidad Europea Miguel de Cervantes. Valladolid.

$\underline{\text { mmatellanes@uemc.es }}$
\end{abstract}

\section{RESUMEN}

La actual Revolución Digital ha planteado un nuevo escenario mundial, en el cual los avances tecnológicos vigentes están diversificando las posibilidades comunicativas; con la aparición de nuevas industrias surgen perfiles profesionales capaces de enfrentar retos de la sociedad del conocimiento.

Los receptores son una pieza clave para lograr la eficacia del mensaje, más aún si hablamos de un contexto actual en el que los canales se modifican -redes sociales- y la relación entre receptores es múltiple y más intensa.

La adecuada segmentación de públicos es sumamente importante si se desea obtener eficacia comunicativa, especialmente en lo relativo a la adecuada gestión de contenidos y mensajes.

Este artículo de investigación tiene por objetivo conocer los usos de Internet según diferentes estilos generacionales, fundamentalmente entre jóvenes de 17 y 30 años. Para ello se han tomado datos estadísticos y fuentes de información secundarias de los años 2009 y 2010 principalmente. Se han revisado los estudios de autores que han estudiado el análisis de los usos de Internet por diferentes generaciones. Además, se ha seleccionado una muestra de 200 estudiantes universitarios de Ciencias de la información de una comunidad universitaria pública y de una comunidad universitaria privada, ambas con sede en Valladolid.

\section{PALABRAS CLAVE}

Internet - Redes Sociales - Grupos Generacionales - Segmentación - Perfil 2.0. 


\section{ABSTRACT}

Actual digital revolution set out a new world scene, where old technological advances are making new possibilities of communication with modern industries with new professional profiles that can face challenges in the knowledge society.

In our context where channels are changing, social networking, and the relationship among recipients are more intense, these recipients are a key piece to achieve an efficacy message.

A good public segmentation is very important if we desire to obtain efficacy communicative and if we want to management contents and messages well. Social

This research has as a main objective knowing different uses of Internet by different generations in a sample of young people between 17 and 30 years. For that it takes statiscal information and second sources of information of 2009 and 2010 years. It takes studies of different authors who have studied analysis of Internet in these subjects. Besides, it selected a sample of 200 university students of Information Sciences in two Universities communities: one of them a public university and another private university. Both of them with head office in Valladolid.

\section{KEY WORDS}

Internet - Social networks - Generations' Groups - Segmentation - Profile 2.0.

\section{ÍNDICE}

$\boldsymbol{1}$ 1. Introducción

$\boldsymbol{N}$ 2. Metodología aplicada

A 3. Resultados

D 3.1 Usuarios de Internet

3.2 Tapscott y la Net-Generación

3.3 Prensky y sus nativos digitales versus inmigrantes digitales

3.4 Oblinger y Oblinger

3.5 Comunidad Universitaria UEMC y UVA

4. Conclusiones y discusión de resultados

5. Bibliografía

\section{INTRODUCCIÓN}

En el ámbito corporativo no sólo ha habido una gran influencia de las nuevas tecnologías en todas las fases del proceso comunicativo (obtención, manejo, 
almacenamiento y difusión de la información); sino que ha cambiado la mentalidad de los estrategas, gerentes y directores de las organizaciones con respecto a: la estructuración de los contenidos, la organización de los procesos operativos y los productos y/o servicios que se ofrecen a los públicos objetivos. Así como también, la automatización de las tareas cotidianas que se hace a través de las nuevas tecnologías ha generado beneficios como el incremento de las oportunidades de negocio, la optimización del tiempo de trabajo, la disminución de los costes de producción y el aumento de la rentabilidad de las empresas (Orihuela, 2008: 57-62).

En este contexto de permanente innovación tecnológica los nuevos medios van apareciendo gradualmente en base a la evolución de los anteriores; es decir, los medios no desaparecen ni son sustituidos sino que tienden a transformarse para poder convivir juntos. Sin embargo, es importante señalar, que sólo sobrevivieron aquellos capaces de redefinir su propia esencia y de adaptarse a los nuevos usos y lenguajes de la realidad digital.

Por ejemplo, la prensa tradicional ha tenido que reinventarse para poder adecuarse al formato digital de Internet; modificando la forma de redactar las noticias y de interactuar con sus lectores. Lo mismo sucede con las redes sociales, especialmente "Twitter" y "Facebook" que han creado nuevos lenguajes y formas de comunicarse.

En consecuencia, según Fumero y García (2008: 65) los medios se han integrado para potenciar su poder comunicativo. La convergencia tecnológica permite que en un mismo dispositivo se puedan unir dos o más medios para complementarse. Tal es el caso de la telefonía móvil que permite a las personas conectarse en Internet, escuchar radio, acceder a redes sociales, tomar fotos, chatear con otros usuarios, enviar mensajes de texto (sms) y; en algunos casos, hasta se puede ver televisión a través del celular. De igual manera, se ha producido una segmentación o fragmentación de la audiencia, lo que conlleva a una especialización en los contenidos para poder satisfacer la demanda y 
adecuarse a las necesidades de los consumidores. Asimismo, se define un perfil de usuario más interactivo y participativo en el cual la persona deja de recibir pasivamente la información y asume un papel mucho más activo.

Esto impulsa el surgimiento de nuevos perfiles que se adapten a los nuevos medios y a las exigencias de públicos claves. Estos profesionales deben tener una visión más integral para que puedan asumir competencias que hasta hace poco eran desempeñadas por otras carreras. Las personas que ocupen estos cargos deberán poseer las siguientes características: estar dotados de una formación cultural amplia, tener una sólida formación académica, conocer a fondo los soportes y las plataformas tecnológicas que se van a utilizar; y ser versátil, creativo e innovador. En los últimos años aparece el nuevo rol del "community manager", que se denomina a la persona que en la empresa está encargada de dinamizar a las redes sociales; teniendo como tarea la gestión de las comunicaciones que están presentes en una comunidad on-line o virtual (Oppermann, 2000: 112-113).

Este nuevo profesional, es el enlace entre la empresa y la sociedad en el contexto de Internet; y tiene como principales actividades: el fomento del diálogo, el incentivar conversaciones que generen valor, motivar debates, identificar líderes de opinión, monitorear el entorno y posicionar a la marca en Internet (Henry, 2006: 25).

En la última década la revolución y notable desarrollo de Internet ha influenciado en los procesos de comunicación y generación e intercambio de información a través de la red. La evolución de la nueva Web 2.0 es evidente y son cada vez más los usuarios que reafirman su uso en las nuevas redes sociales, especialmente intenso en el caso de los españoles, aunque con un escaso grado de tecnificación (Eroski Consumer, 2010). Esta nueva perspectiva nos acerca a diferentes paradigmas comunicacionales que determinan la interacción entre diversos usuarios que navegan por la red. 
La facilidad de acceso y de uso en Internet se destaca en los últimos años, lo que facilita el crecimiento de uso de las nuevas redes sociales. Un estudio realizado por Zed Digital (2008), señala que el 50\% de los internautas son usuarios habituales de dichas redes. Según el informe, dos de cada tres reconocen que las utilizan para realizar recomendaciones sobre marcas.

En este contexto se consolida la conocida Web 2.0, siendo las redes sociales, el máximo exponente de un fenómeno que ha trascendido a los primeros usos informáticos en los años noventa. Las redes sociales son un fenómeno flamante y clave para entender los próximos modelos de comunicación interpersonal.

\section{METODOLOGÍA APLICADA}

A través de esta investigación se plantea analizar el impacto del medio Internet entre diferentes tipologías de usuarios. Sin embargo, debido a la magnitud del objeto de estudio, se ha centrado en el perfil dominante en términos de volumen: jóvenes de entre 17 y 30 años que serán analizados desde una perspectiva multidisciplinar contemplando aspectos sociológicos, tecnológicos y, además culturales. De este modo, con el estudio particular de dichos individuos será más fácil para el rol del "community manager" segmentar de un modo más eficiente.

Para ello se parte de la hipótesis de que la aparición de Internet y, con ella su uso, ha dado lugar cambios importantes en las pautas de comportamiento desde el punto de vista sociológico, cultural y tecnológico entre los usuarios más avanzados o aquellos que ya poseen una experiencia derivada de los más de quince años de uso extendido en España. Se ha planteado una base metodológica basada en la triangulación.

Cuando se habla de triangulación desde el punto de vista de Oppermann, cabe destacar que hay que hacer referencia a la utilización de diversos tipos de métodos, perspectivas o datos en una misma investigación de un problema. Se han empleado datos estadísticos que cuantifican el volumen de usuarios. 
Asimismo, se han analizado diferentes perspectivas que se están utilizando para clasificar al usuario y se han señalado las prácticas y usos dominantes para, finalmente concluir con algunas consideraciones finales.

Con el fin de conocer el uso cotidiano y la estructura de las redes sociales en el ámbito universitario, se seleccionó el universo de estudiantes de dos comunidades universitarias, la Universidad Europea Miguel de Cervantes, de titularidad privada y con sede en Valladolid y la Universidad de Valladolid, de titularidad pública y con sede también en Valladolid. De esta forma, se podrían comparar los resultados desde el punto de vista de los estudiantes de Universidad pública y por otro lado, los de Universidad privada.

El método de obtención de información fue la encuesta estructurada. En dicha encuesta se utilizaron esencialmente preguntas dicotómicas, cerradas y basadas en escalas. Se determinó el uso de escalas impares, de tipo acuerdo desacuerdo en cinco posiciones, posibilitando la neutralidad en la opinión, ya que al ser un estudio a priori interesante para los encuestados se esperó una adecuada respuesta.

En cuanto a la técnica de muestreo, se utilizó el muestreo por cuotas. Se trataba de asegurar la adecuada representatividad del universo, por lo que se reprodujo en dicho muestreo la estructura del universo de estudio en razón de sexo y edad en primer nivel. Los estudiantes de ambas universidades son de las licenciaturas de Ciencias de la Información con edades comprendidas entre los 17 y 30 años.

El procedimiento de investigación se desarrolló durante el curso lectivo 20092010. El cuestionario contiene 8 preguntas cerradas, referentes a cuestiones de conocimiento en cuanto a frecuencia, uso, estructura, preferencia y opinión. 
Tabla 1. Cuestionario utilizado

\begin{tabular}{|l|l|}
\hline Ámbito & Castilla y León / Valladolid / UEMC y UVA. \\
\hline Técnica & Cuestionario autoadministrado. \\
\hline Población & 26.500 Estudiantes. \\
\hline Elementos de la muestra & Estratificada y cuotada. Muestra 200 sujetos. \\
\hline
\end{tabular}

Para la explotación de datos se utilizó el software específico SPSS (Stadistical Package for Social Sciences) v. 16.

\section{RESULTADOS}

\subsection{Usuarios de Internet}

Al hablar de usuarios de Internet hay que partir de los datos estadísticos que permitan cuantificar la magnitud del objeto de estudio, así como conocer cuál es el perfil sociodemográfico clave. Para tal fin se han utilizado los datos del informe realizado por el Observatorio Nacional de las Telecomunicaciones y de la Sociedad de la Información para 2010 (www.ontsi.red.es).

Según el citado informe, el número de usuarios de Internet de más de diez años ha alcanzado casi los veintitrés millones y medio de individuos (23.471.120), es decir un 9,8 \% más que en 2009. Para entender gráficamente la importancia de esta cifra se ha comparado con el volumen total de población en España correspondiente al mismo perfil de edad, es decir, de entre 10 y 77 años. Siguiendo los datos del Instituto Nacional de Estadística, se observa que el número de personas para enero de 2010 con estas características es de 38.379.616 personas, por tanto se puede concluir que el 61,1 \% de este grupo de población es usuario de Internet en España.

Figura 1. Porcentaje de usuarios de Internet de entre 10 y 77 años 


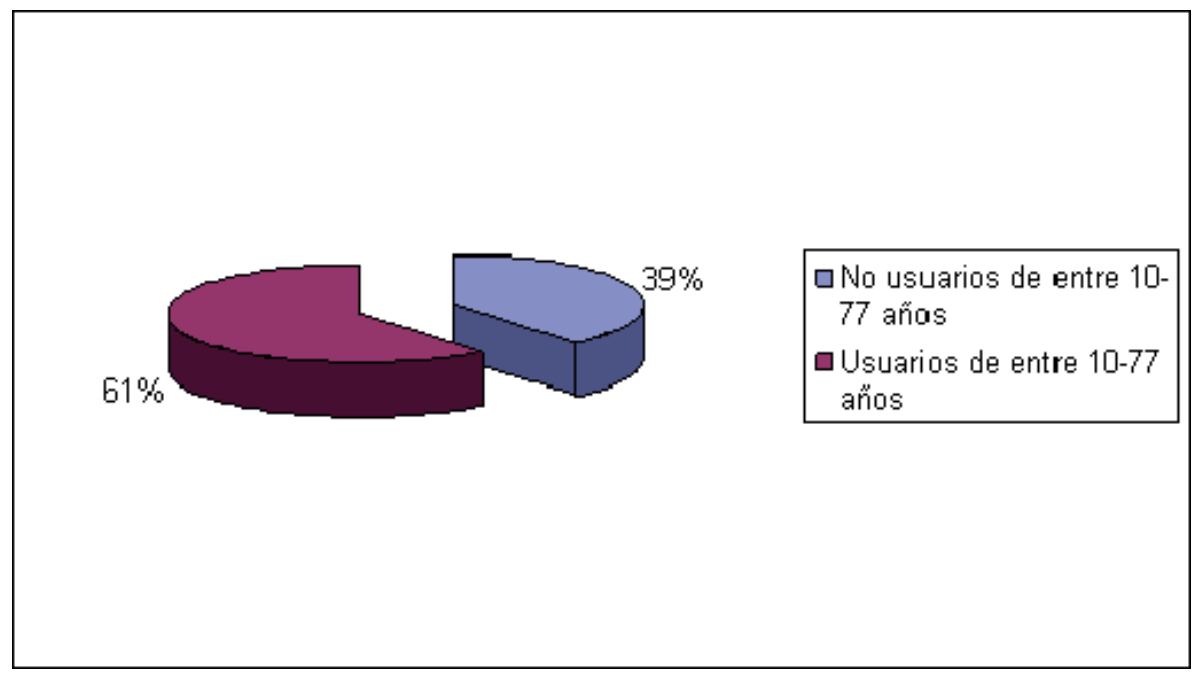

Fuente: elaboración propia a partir de los datos del Ine

Por otro lado, este dato estadístico reflejado en el gráfico, muestra una realidad que se viene observando desde hace años: el crecimiento exponencial de los usuarios de Internet. Y es que cada día van llegando nuevos visitantes a la Red que van adoptando y haciendo suyas prácticas y usos que los más avanzados y expertos han difundido y, por tanto, contribuyen con su uso y experiencia a la difusión de las tecnologías de distribución que caracterizan a este medio y, lo que es más importante, están contribuyendo a la difusión de los protocolos y prácticas culturales que se corresponden con el segundo nivel de Internet como medio. En este crecimiento exponencial convergen diferentes factores como la expansión de los recursos tecnológicos, el posicionamiento alcanzado de los diferentes usos de la Red tales como las redes sociales, los blogs, los podcast y sobre todo, la aceptación y el reconocimiento generalizado de la utilidad de este medio. Respecto a este último factor, y como ya se ha señalado anteriormente, una vez que un medio se establece satisfaciendo alguna exigencia humana fundamental, continua funcionando dentro de un vasto sistema de opciones mediáticas, que en el siglo XXI, aparecen integrados dentro del paradigma de la convergencia.

Un perfil sociodemográfico dominante de usuario en Internet, corresponde a hombres de entre 16 y 44 años de edad (según el informe de la Ontsi de 2010) en 
situación laboral activo ocupado y con estudios universitarios o de segunda etapa de educación secundaria. Sin embargo, conviene señalar que, aunque este es el perfil dominante, los mayores de 44 años cada vez están más representados en las estadísticas lo que reafirma la idea de que cada día van llegando nuevos usuarios, nuevos consumidores, productores y difusores del contenido multimedia a Internet tanto de las ciudades como de los municipios, que en anteriores informes, estaban menos representados. Respecto a la frecuencia, prácticamente el $81,8 \%$ de los usuarios de entre 16 y 24 años acceden a Internet semanalmente o diariamente. Este dato muestra que este segmento poblacional ha hecho ya suyo el medio y está totalmente identificado con el uso de él. De hecho siguiendo el 49,4\% son usuarios de Internet desde hace más de ocho años (www.aimc.es). Asimismo, el 67,6\% de los usuarios que tienen entre 25 y 34 años acceden también con una frecuencia semanal o diaria a la red. De esta forma se comprueba que el grueso de usuarios familiarizados con la Web se corresponde a un grupo de entre 16 y 34 años de edad, ya que la frecuencia de uso muestra que este medio ya forma parte de su día a día. Por tanto, con estas cifras se ha identificado el perfil dominante por edad del usuario que utiliza este medio y que con su uso está contribuyendo a la exploración de nuevas líneas que permitan superar la incertidumbre que actualmente rodea a Internet.

\subsection{Tapscott y la Net-Generación}

Don Tapscott (1998: 45-47) en "Growing up digital: The rise of the Net Generation" hace una primera clasificación dicotómica basada en el impacto de la tecnología y su uso en la población estadounidense. En esta obra el autor define dos grupos generacionales marcados por el contraste y el impacto de la tecnología dominante. De esta forma se destaca un primer grupo generacional denominado "Net Generation", "N-Generation”, o "N-Geners", jóvenes nacidos entre los años 1977 y 1997 que en el momento de publicación del libro tenían 
entre los dos y los veintidós años de edad. Lo realmente significativo de esta primera clasificación realizada hace diez años es que el autor utiliza una terminología que extiende a toda una generación que en el momento de publicación del libro no eran usuarios activos de Internet. Así, anteponiéndose al devenir, Tapscott ya preveía una serie de cambios generacionales basados en el impacto de las tecnologías de distribución y en el aumento significativo del volumen de otra generación venidera, es decir, eran las primeras generaciones que habían superado en número a los nacidos entre 1946 y 1964, los llamados "Baby Boomers", representando así el 30\% de la población frente al 29\% correspondiente a estos últimos. Estos jóvenes definidos por el autor tienen ahora de 12 a 32 años con lo que coinciden a grandes rasgos con el perfil de usuario dominante en España mientras que, la generación de los “Baby Boomers" tendría hoy día entre 45 y 63 años de edad.

\subsection{Prensky y sus nativos digitales versus inmigrantes digitales}

Dos años más tarde aparece otra clasificación dicotómica con características muy parecidas a la anterior, pero con tintes algo más radicales. Se trata de Marc Prensky y su expresión "Digital Natives" que lanza en oposición a "Digital Inmigrants", los inmigrantes llegados tarde a las Tecnologías de la Información y Comunicación, en un ensayo publicado en 2001 bajo el título: "The death of command and control" (Tapscott, 1998: 112). Cabe destacar que la motivación que lleva a Prensky a utilizar estas expresiones tiene su origen en la necesidad de abordar un inquietante problema derivado del cambio generacional: la crisis de la educación. Prensky (2010: 114-115) define a los llamados inmigrantes digitales como aquellos que utilizan la tecnología, conviven con ella, disfrutan de ella pero nacieron y crecieron sin ella.

Frente a ellos estarían los nativos digitales que sólo han conocido una sociedad dominada por el uso de las nuevas tecnologías. Asimismo, se señala que una de 
las principales diferencias entre ambos es el mantenimiento de cierta conexión de los inmigrantes digitales con su entorno previo. En este sentido, resulta realmente significativo el término que acuña Prensky como acento del inmigrante digital. Según el autor la experiencia práctica de este término se puede apreciar en diferentes aspectos tales como la necesidad de impresión de un e-mail o la impresión de un documento escrito en el ordenador para corregirlo ante la dificultad visual de hacerlo en la pantalla pero sin embargo, se considera que lo que Prensky denomina como acento del inmigrante digital, también puede ser extendido a los colectivos más jóvenes como se verá más adelante al hablar de una clasificación del usuario en función de la experiencia y de los conocimientos aprendidos (Tapscott, 1998: 129). Respecto a las características de los nativos digitales, se puede señalar, entre otras, la rápida recepción de la información, facilidad para los procesos y multitareas paralelos, predilección por la imagen antes que el texto, defensa y práctica de la navegación no secuencial y la capacidad de cooperación y predisposición al trabajo en red. Teniendo en cuenta el objeto de estudio, lo verdaderamente significativo de este autor es que apoya su tesis en argumentos neurobiológicos, argumentando y difundiendo tesis como la de Bruce D. Perry, según la cual diferentes experiencias conducen a diferentes estructuras cerebrales. De esta forma Prensky (2010: 101-102) hace referencia a las investigaciones y teorías científicas relacionadas con la neuroplasticidad del cerebro donde se pone de manifiesto que la influencia de las experiencias vividas tiene implicaciones en los patrones de pensamiento.

En este sentido argumenta que las actividades desarrolladas en entornos virtuales de forma continuada, como en el caso de los nativos digitales con los videojuegos, tienen como consecuencia un desarrollo mayor de habilidades como la interpretación de imágenes visuales, representaciones tridimensionales, mapas mentales, la representación mental de combinaciones de formas, el descubrimiento inductivo, la atención simultánea sobre distintos focos.., lo que 
reafirma la hipótesis inicial del impacto de la tecnología sobre los usuarios experimentados. Para Prensky (2010: 89-90), los nativos digitales prefieren las imágenes al texto, también están acostumbrados a un ritmo frenético de información y se sienten cómodos trabajando en multitarea, además, están acostumbrados a un mundo con sobrecarga informativa y ritmo frenético ("twitch speedy") y anteponen las gratificaciones instantáneas y las recompensas inmediatas a las de largo plazo. Por último, es conveniente profundizar en los cambios neurobiológicos que ya citó Prensky y que se ampliarán con las investigaciones del Doctor Garry Small durante el año 2009, quien señala que aunque pueda parecer que la exposición a las nuevas tecnologías produce un impacto mucho más sutil, sus efectos estructurales y funcionales son profundos, en particular en el caso del cerebro joven, extremadamente plástico. Así, el bombardeo de estímulos digitales sobre las mentes en desarrollo de los nativos digitales está enseñando a reaccionar más deprisa y a codificar la información de un modo diferente. Y es que según las investigaciones de Small (2009: 89-92), la tecnología digital, además de influir en cómo pensamos, está cambiando la forma de sentir y de actuar, y el modo de funcionar el cerebro. Para el autor, la mente joven es como un ordenador nuevo que lleva incorporados algunos programas básicos y dispone de muchísimo espacio en el disco duro para información adicional. Por tanto, Small señala que, a medida que van entrando más y más datos en la memoria del ordenador, éste va creando atajos para acceder a la información y éstos quedan asentados como nuevos caminos neuronales. La inmensa cantidad de posibles conexiones viables explica la plasticidad del cerebro joven, su maleabilidad y su capacidad de cambio permanente en respuesta a los estímulos que le llegan del entorno. Por tanto, siguiendo a este autor, la resolución de la alta tecnología ha sumido en un estado de atención parcial continua que Linda Stone, una autoridad en software, describe como estar permanentemente ocupados, siempre atentos a todo, pero sin centrarse nunca de verdad en algo concreto. Así, en Small se 
destaca una precisión del término empleado por Prensky donde señala que la atención parcial continua es distinta de la multitarea, pues en este sentido se tiene un objetivo para cada tarea, y se intenta mejorar la eficacia y la productividad. En cambio, cuando la mente atiende de forma parcial, y lo hace así de manera continuada, se busca la oportunidad de cualquier tipo de contacto en todo momento (Small, 2009: 102-103).

\subsection{Oblinger y Oblinger}

Oblinger y Oblinger (2005: 78-79) continúan en la línea de Tapscott y Prensky definiendo a estos jóvenes, pero sin embargo introduce una matización importante con la necesidad de considerar que las características que se les atribuye a los jóvenes no tienen su origen en un rango de edad sino en unas experiencias concretas. Así se discurre sobre una nueva aportación que matizaría la clasificación generacional anterior, dando paso a una flexibilidad en la que la experiencia es la base. Y es que muchas de las actitudes y rutinas atribuidas a la "Net Generation" se pueden observar igualmente en personas de más edad, pero que usan de forma cotidiana estas tecnologías. Así, acceden de forma prioritaria a la información a través de Internet, realizan varias tareas en paralelo y tienen una conexión a la red casi permanente.

Se habla de los jóvenes como personas optimistas y resueltas, aficionadas a las nuevas tecnologías, pero también de activistas decididos a implicarse públicamente. Pero nuevamente los autores van más allá definiendo características negativas asociadas a los jóvenes ya que tendrán una reacción negativa con cualquier contenido o actividad que les parezca lenta (Fernández, 2008: 118).

Roca (2008: 76) describe a estos jóvenes como un perfil fascinado por las nuevas tecnologías y con una enorme facilidad para desenvolverse con ellas. Estos 
jóvenes y adolescentes han integrado entornos virtuales como la mensajería instantánea en su forma de relacionarse con los demás, hasta el punto de no establecer una barrera clara entre los contactos a través de la red y los contactos presenciales. También tienen una preferencia clara por las imágenes frente al texto, una habilidad innata para la orientación espacio-visual fruto de su experiencia con juegos digitales, una clara tendencia hacia el aprendizaje a través de la exploración y una sorprendente capacidad para mantener la atención sobre varios focos de forma simultánea. Unas características que entroncan con las definidas por Prensky.

\subsection{Comunidad Universitaria de la UEMC y la UVA}

No se han detectado diferencias importantes entre los resultados de los estudiantes pertenecientes a la Uva (comunidad universitaria pública) frente a los estudiantes de la Uemc (comunidad universitaria privada). Asimismo, tampoco se han detectado importantes diferencias en la variable sexo, destacando que el mayor porcentaje de encuestados fueron mujeres, un $72 \%$ del total de la muestra de 200 estudiantes.

Respecto al tiempo o frecuencia de uso a la semana, el mínimo de conexión en la Uva se sitúa entre una y dos horas, con un $44 \%$ de representación, entre dos y cuatro horas con un $30 \%$ y entre cuatro y ocho horas con un $23 \%$.

En el caso de la Uemc es de entre una hora y dos horas, con un $49 \%$, entre dos y cuatro horas con un $33 \%$ y entre cuatro y ocho horas con un $18 \%$.

Estas cifras demuestran que Internet es un medio que se ha asentado como entretenimiento ya que su uso se relaciona con el hogar y presenta una frecuencia de tiempo de dedicación de más de una hora, llegando a situarse incluso en más de ocho horas. Se supone que el aumento de la frecuencia y uso de Internet se ha hecho en detrimento de otras actividades. 
Los estudiantes de la Uva usan preferentemente Internet para la búsqueda de información para la realización de trabajos y estudios con un 32\%, un 39\% como forma de contacto a través del uso de las redes sociales, un 15\% para ver televisión y un 14\% para otros usos no identificados. Los resultados de la Uemc son muy similares: un $29 \%$ para estudios, un $43 \%$ como modo de contacto en redes sociales y un $28 \%$ para otros usos, identificando preferentemente los juegos digitales.

En otras de las preguntas se cuestionaba la preferencia de uso de Internet para ver televisión. Muchos de los encuestados declaran que el tiempo empleado en la Red ha repercutido en la visualización de la televisión con un 35\% en la Uva y un $29 \%$ en la Uemc. La lectura también se ha visto afectada.

Es importante destacar que tanto los estudiantes de la Uva como de la Uemc usa Internet como entretenimiento y como instrumento de comunicación con sus amigos y familiares. Es un colectivo que, a diferencia de los usuarios de edades más avanzadas, utiliza los videos online, los videojuegos por ordenador y mundos virtuales como una forma más de entretenimiento.

En este sentido, cabe destacar que el uso de los videojuegos y juegos online se está convirtiendo en una de las prácticas estrellas para los jóvenes en general y, los usuarios de entre 12 y 17 años en particular, ya que 78\% utilizan la red para juegos en línea (datos del Informe Aimc de 2010).

Por otro lado, la consulta del correo sigue liderando las posiciones con un $80 \%$ y 85\% en la Uva y Uemc respectivamente.

Los usuarios de Internet de entre 17 y 29 años principalmente, leen los blog y escriben en uno propio, a la vez participan en las redes sociales con mucha más frecuencia que las de las poblaciones más avanzadas en edad. Además, nuevamente en contraste con las poblaciones más maduras, el uso de la mensajería instantánea se ha convertido en algo habitual para este colectivo. 
Enlazando con los datos del informe Aimc de 2010, en contraste con la llamada Generación Y, los usuarios de entre 32 y los 72 años, utilizan la Red como una herramienta de búsqueda de información, envío de e-mails y compra de productos más que como una red de socialización y de entretenimiento. Aunque en este sentido conviene señalar que, siguiendo los datos de los encuestados de dicho informe de 2010, muchos de ellos más del 50\%, declaran que Internet es la fuente primaria a la que acceden para informarse y un 41,5\% la utilizan como fuente secundaria.

Llama la atención el bajo grado de interés que despiertan las compras online en las poblaciones más jóvenes, únicamente un 22\% de ellos en la Uva y un 15\% de la Uemc. Esto tiene mucho que ver con el poder adquisitivo de los estudiantes que en las circunstancias dadas es bajo en muchos de los casos.

En definitiva, si se tienen en cuenta prácticas comunes en los diferentes perfiles de usuarios clasificados por edad, cabe destacar la consulta de trabajos de investigación, contacto a través de redes sociales, descarga de vídeos y las reservas de viajes por Internet.

\section{CONCLUSIONES Y DISCUSIÓN DE RESULTADOS}

Parece pertinente afirmar que el usuario 2.0 posee un perfil avanzado, activo e interactivo que no sólo consume las posibilidades que ofrece la red sino que las explora y las difunde al mismo tiempo que utiliza las redes sociales virtuales como punto de encuentro estableciendo un nuevo modelo de sociabilidad en el que el uso de las tecnologías de distribución está creando nuevas líneas de protocolos y de comportamientos definidos en el marco de la convergencia comunicativa (Small, 2009: 25).

Así, esta definición podría conducir a hablar también de una generación 2.0, de un perfil 2.0, de una actitud 2.0 o incluso de una personalidad 2.0. Porque lo cierto, es que quien se enfrenta a las nuevas aplicaciones online ya no es un 
espectador pasivo, ahora es un usuario con unas características muy peculiares, con hábitos adquiridos y conocimientos demostrados.

Continuando con la clasificación de los usuarios, desde un punto de vista económico, hay que señalar la utilizada en las investigaciones llevadas a cabo por la "Pew Research Center's Internet \& American Life Project surveys", donde se distingue una clasificación más personalizada de los diferentes perfiles o grupos de usuarios encuadrados en el marco geográfico de los Estados Unidos.

En este estudio la población de usuarios ha sido clasificada y nombrada en función de la edad lo que deriva en una clasificación generacional. Sin embargo, se considera que es una categorización centrada en intereses económicos, ya que la edad mínima cuantificada corresponde a los 18 años con lo que estaría íntimamente relacionado con la capacidad adquisitiva de los individuos.

Así, se destacan seis grupos generacionales que, en orden ascendente, comenzaría con la llamada Generación Y, usuarios de edades comprendidas entre los 18 y los 32 años. Seguidamente encontramos a la Generación X, personas de 33 a 44 años de edad. "Youngers Boomers" se refiere a las poblaciones de usuarios de 45 a 54 años. "Older Boomers" hace referencia a la población de 55 a 63 años. Y para aquellos que se encuentran entre los 64 y los 72 años se ha utilizado el término "Silent Generation".

Por último estaría la Generación I que hace referencia a los usuarios de más de 73 años de edad. De esta clasificación se puede señalar que por norma general, como ya se ha dicho anteriormente, se suele distinguir seis perfiles de edad con su correspondiente modo de comportamiento. En este sentido, no olvidando el objeto de investigación original, se puede concluir que el perfil dominante formaría parte de la llamada Generación X y de la Generación Y.

Por otro lado, respecto al comportamiento del usuario, y muy relacionada con la clasificación en función de la experiencia, en la Web se distinguen cuatro actitudes: pasivo, participativo, cooperativo y/o colaborativo. 
Figura 2. Clasificación del usuario respecto a su comportamiento

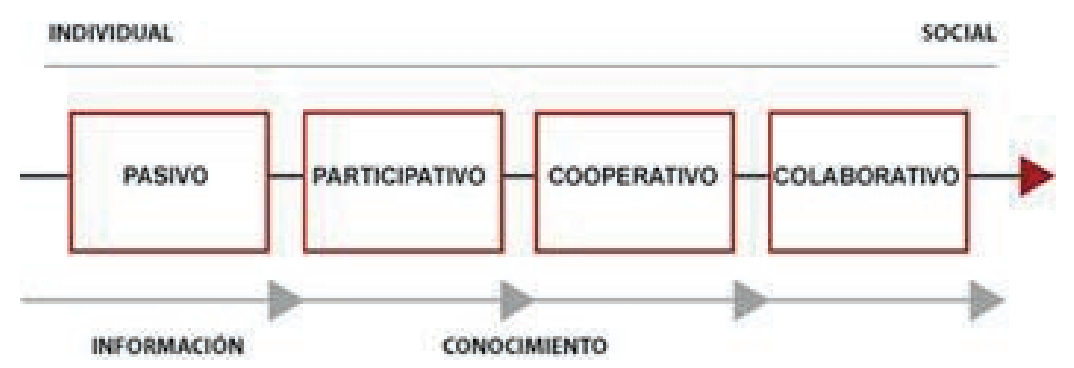

Fuente: Yusef (2007: 55)

Como se puede comprobar en el gráfico de Yusef (2007: 55), se observa un primer comportamiento denominado pasivo. Este término hace referencia al usuario que únicamente consume la información sin derivarla en conocimiento. En esta línea cabe destacar que vivimos en una época donde la información es el principal bien de consumo y si esa información no deriva en conocimiento, el derecho que consagra la Constitución española se desintegraría. Y es aquí donde entra el nuevo perfil de consumidor de la información o, como diría Yusef de usuario 2.0. En este nuevo perfil se distinguen distintos niveles de comportamiento en función de la experiencia. De este modo, se puede encontrar a un usuario participativo que derivará en cooperativo y colaborativo, no siendo lineal esta evolución.

Si bien es cierto que determinados factores, como en el caso del profundo cambio tecnológico que está suponiendo la llegada de Internet, sí pueden crear saltos generacionales más importantes que los derivados de las habituales y previsibles divergencias entre jóvenes y mayores, aún considerando que estos cambios en las circunstancias que acompañan el desarrollo personal pueden afectar a las maneras de aprender y pensar, existe un excesivo determinismo tecnológico en el discurso de quienes han defendido que los jóvenes que han nacido en un mundo digital tienen una forma de comportarse radicalmente diferente. 
Los usuarios al acceder a Internet, ya sean Nativos o Inmigrantes Digitales, lo hacen motivados por la búsqueda de un producto que satisfaga sus necesidades o deseos, es decir dicho con otras palabras buscan la utilidad, que según señala Yusef Hassan-Montoro (2009: 45-47), es una utilidad entendida como el provecho, beneficio e interés que produce su uso. Cuando un usuario se enfrenta a una interfaz, será capaz de identificar y reconocer la función de cada elemento sólo si ha aprendido previamente su significado, o ha adquirido las herramientas conceptuales necesarias para deducirlo. Esto quiere decir que el uso de interfaces son actividades sociales, aún cuando se realicen de forma aislada, pues se basan en significados compartidos y construidos colectivamente.

Tras el análisis realizado se puede señalar que el perfil dominante por edad del usuario de Internet se corresponde a los jóvenes de entre 16 y 34 años de edad. Se comprueba que cada día van llegando nuevos visitantes a la red que van adoptando y haciendo suyas prácticas y usos que los más avanzados y expertos han difundido y, por tanto, contribuyen con su uso y experiencia a la difusión de las tecnologías de distribución que caracterizan a este medio y, lo que es más importante, están contribuyendo a la difusión de los protocolos y prácticas culturales que se corresponden con el segundo nivel que define a Internet como medio. Y es que la capacidad de participación no procede de destruir la cultura sino de escribir sobre ella, modificarla, corregirla, expandirla, conferirle una mayor diversidad de perspectiva y luego volver a ponerla en circulación, reintroduciéndola en los medios dominantes para su libre circulación.

En este crecimiento exponencial convergen diferentes factores como la expansión de los recursos tecnológicos, el posicionamiento alcanzado de los diferentes usos de la red tales como las redes sociales, los blogs, los podcast y sobre todo, la aceptación y el reconocimiento generalizado de la utilidad de este medio. Respecto a este último factor se puede concluir que Internet es un medio 
que se está estableciendo satisfaciendo exigencias humanas básicas como se comprueba con la frecuencia de uso y del volumen de los usuarios. Por tanto, gracias a la satisfacción de necesidades, parece pertinente afirmar que continuará funcionando dentro de un vasto sistema de opciones mediáticas, que en el siglo XXI, aparecen integradas dentro del paradigma de la convergencia.

Tras el análisis de las diferentes clasificaciones de usuario por grupos generacionales, se ha comprobado que sigue predominando cierto determinismo tecnológico derivado de una segmentación dicotómica que focaliza la atención en el impacto tecnológico y las diferencias generacionales marcadas por el volumen de la muestra a analizar y el medio dominante.

Esta brecha generacional parece respaldada por las investigaciones neurobiológicas donde se señala que el bombardeo de estímulos digitales sobre las mentes en desarrollo está enseñando a reaccionar más deprisa y a codificar la información de un modo diferente al mismo tiempo que se está produciendo una pérdida de atención. Así, las actividades desarrolladas en entornos virtuales de forma continuada tienen como consecuencia un desarrollo mayor de habilidades como la interpretación de imágenes visuales, representaciones tridimensionales, mapas mentales, la representación mental de combinaciones de formas, el descubrimiento inductivo y la atención simultánea sobre distintos focos. En este sentido, se considera válida una clasificación dicotómica por grupos generacionales contemplando el hecho de la mayor predisposición del cerebro joven por su mayor plasticidad sin embargo, también se podría hacer extensible este impacto a las poblaciones adultas ya que, según se ha indicado, la clasificación por experiencia parece la más acertada. De esta clasificación por experiencia se deriva una serie de conceptos como lo son: usuario 2.0, personalidad 2.0 o perfil 2.0 que hacen referencia al grado de asimilación e implantación de Internet entre la población. 
En el caso de las comunidades universitarias estudiadas, no se han apreciado diferencias significativas entre el carácter universitario de tipo público o privado.

Lo que sí es cierto es que ambas tipologías de estudiantes corresponden a los llamados nativos digitales que han nacido en la era de Internet, considerando ya a este medio como un "comodity" dentro de la sociedad actual.

\section{BIBLIOGRAFÍA}

- AIMC: "Navegantes en la Red: $12^{\circ}$ encuesta AIMC a usuarios de Internet". Madrid, 2010.

- AIMC: "Navegantes en la Red: $11^{\circ}$ encuesta AIMC a usuarios de Internet". Madrid, 2009.

- EROSKI CONSUMER: "Un estudio sitúa a los españoles entre los europeos más activos en redes sociales". 2010. Disponible en http://www.consumer.es/

- FERNÁNDEZ, S.: "Redes Sociales. Fenómeno pasajero o reflejo del nuevo internauta". Telos, 76, p. 118, Madrid, 2008.

- FUMERO, A. y GARCÍA, M.: "Redes Sociales. Contextualización de un fenómeno dos-punto-cero". Telos, 76, p. 65, Madrid, 2008.

- HASSAN-MONTERO, Y.: “Evolución del perfil del usuario. Usuarios 2.0.". 2007. Disponible en: http://www.monografias.com/trabajos78/jovenesmedios-digitales/jovenes-medios-digitales2.shtml).

- HASSAN-MONTERO, Y., ORTEGA-SANTAMARÍA, S.: "Informe APEI sobre Usabilidad". Asociación Profesional e Especialistas en Información, Gijón, 2009.

- HENRY, J.: “Convergent Culture". New York University, 2009. 
- JONES, S.: "Generations online in 2009. Pew Research Center's Internet $\mathcal{E}$ American Life Project surveys". 2009. Disponible en: (http://pewresearch.org/pubs/1093/generations-online).

- MCLUHAN, M.: “Understanding Media: The extensions of man". Londres, 2001.

- OBLINGER, D. G. Y OBLINGER, J. L.: “Educating the Net Generation”. Oxford (UK), 2005.

- ONTSI: "Informe del Observatorio nacional de las telecomunicaciones y de la sociedad de la información". Madrid, 2009.

- OPPERMANN, M.: "Triangulation - A Methodological discussion. International Journal of Tourism Research". Londres, 2000.

- ORIHUELA, J. L.: “Internet: la hora de las redes sociales". Nueva Revista, 119, pp. 57-62, 2009.

- OVER, R., BENITO, M., ROMO, J.: "Nativos digitales y aprendizaje: una aproximación a la evolución de este concepto". Revista Icono 14, pp. 31-53, 2009.

- PRENSKY, M.: “Teaching digital natives”. Corwin, 2010.

- ROCA, G.: "Soluciones para nuevas realidades. Modelos de negocio en las redes sociales". Madrid, Telos, 76, 2008.

- SMALL, G.: “El cerebro digital”. Barcelona, 2009.

- TAPSCOTT, D.: “Growing up Digital: the Rise of the Net Generation". Nueva York, 1998.

- ZED DIGITAL: "Estudio sobre el Fenómeno de las Redes Sociales en España: percepción, usos y publicidad". 2008. Disponible en http:/ / www.zeddigital.es/ 